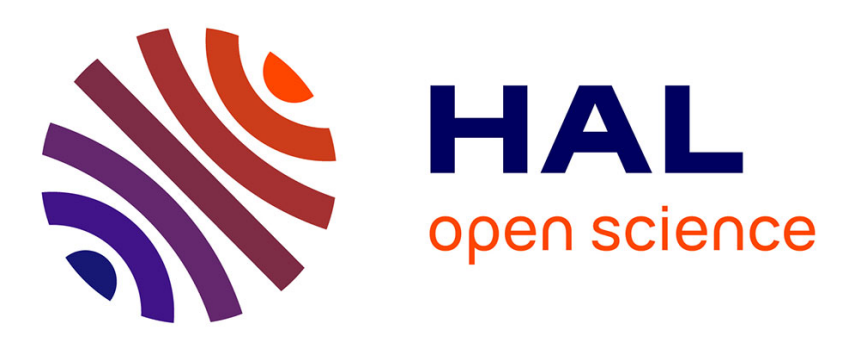

\title{
La production de céramique commune à Pompéi. Un four de potier dans l'insula 5 de la regio I Laetitia Cavassa
}

\section{To cite this version:}

Laetitia Cavassa. La production de céramique commune à Pompéi. Un four de potier dans l'insula 5 de la regio I. Michel Pasqualini. LES CÉRAMIQUES COMMUNES D'ITALIE ET DE NARBONNAISE, Collection du Centre Jean Bérard (30), pp.95-104, 2009. halshs-03037480

\section{HAL Id: halshs-03037480 \\ https://shs.hal.science/halshs-03037480}

Submitted on 3 Dec 2020

HAL is a multi-disciplinary open access archive for the deposit and dissemination of scientific research documents, whether they are published or not. The documents may come from teaching and research institutions in France or abroad, or from public or private research centers.
L'archive ouverte pluridisciplinaire HAL, est destinée au dépôt et à la diffusion de documents scientifiques de niveau recherche, publiés ou non, émanant des établissements d'enseignement et de recherche français ou étrangers, des laboratoires publics ou privés. 
LES CÉRAMIQUES COMMUNES

\section{D'ITALIE ET DE NARBONNAISE} STRUCTURES DE PRODUCTION, TYPOLOGIES ET CONTEXTES INÉDITS II siècle av. J.-C. - III ${ }^{\mathrm{e}}$ siècle ap. J.-C.

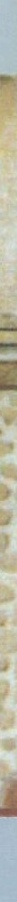


ACR 2003-2006 Action Collective de Recherche Archéologie du territoire national

"Les céramiques communes de Marseille à Gênes»

\section{LES CÉRAMIQUES COMMUNES}

D'ITALIE ET DE NARBONNAISE 
Les céramiques communes d'Italie et de Narbonnaise : structures de production, typologies et contextes inédits, II ${ }^{\mathrm{e}}$ s. av. J.-C.-III ${ }^{\mathrm{e}}$ s. apr. J.-C. : actes de la table ronde de Naples, organisée les 2 et 3 novembre 2006 par l'Action collective de recherche «Archéologie du territoire national» et le Centre Jean Bérard / sous la direction de Michel Pasqualini. — Naples : Centre Jean Bérard, 2009. — 724 p. : ill. ; 30 cm (Collection du Centre Jean Bérard, ISSN 1590-3869 ; 30)

ISBN 978-2-903189-97-6

I. Pasqualini, Michel

Diffusion De Boccard 11 , rue de Médicis 75006 Paris
L'ERMA di Bretschneider Via Cassiodoro, 19 00193 Roma
M. D’Auria Editore Calata Trinità Maggiore,52 80134 Napoli
EDIPUGLIA

Via Dalmazia, 22/B

70050 Bari-S. Spirito

Collection du Centre Jean Bérard, ISSN 1590-3869 ; 30

(C) Centre Jean Bérard - ISBN 978-2-903189-97-6 
Collection du Centre Jean Bérard, 30

\title{
LES CÉRAMIQUES COMMUNES D'ITALIE ET DE NARBONNAISE
}

\author{
STRUCTURES DE PRODUCTION, TYPOLOGIES ET CONTEXTES INÉDITS \\ II ${ }^{\mathrm{e}}$ s. av. J.-C. - III ${ }^{\mathrm{e}}$ s. apr. J.-C.
}

Sous la direction de Michel Pasqualini

Actes de la table ronde de Naples organisée les 2 et 3 novembre 2006 par l'Action Collective de Recherche « Archéologie du territoire national » et le Centre Jean Bérard

publiés avec le concours de :

Ministère de la Culture

Direction de l'architecture et du patrimoine, sous-direction de l'archéologie, de l'ethnologie, de l'inventaire et du système d'information

\section{CNRS}

Cépam / CNRS-UNSA, UMR6130

Centre Jean Bérard, CNRS - École Française de Rome

Institut national de recherches archéologiques préventives

Ville de Fréjus

Direction de la Culture, Service du Patrimoine

Naples 2009 


\section{Remerciements}

Au-delà de tous les camarades qui ont accepté de participer à ce projet, je veux saluer la mémoire de Paul-Albert Février, remercier Michel Bats et Jean-Pierre Brun qui m’ont donné le goût du travail d'équipe et Frank Braemer qui m'a offert de diriger ce travail et m'a encouragé à promouvoir ce partage d'idées et d'expériences, sans oublier mon camarade Michel Bonifay avec qui j'ai partagé la même école.

Merci à Monsieur Xavier Delestre, Conservateur régional d'archéologie de PACA ; Madame Giuseppina Spadea, Surintendant archéologue de Gênes, Madame Maria Luisa Nava, Surintendant archéologue de Naples et Caserta et Monsieur Pietro Giovanni Guzzo, Surintendant archéologue de Pompéi ; Mesdames Herveline Delhumeau et Sophie Goedert, Direction de l'architecture et du patrimoine au Ministère de la Culture et de la Communication; Monsieur Jean-Paul Jacob et Madame Nicole Pot, respectivement Président et Directrice générale de l'Inrap ; Monsieur François Souq, Directeur de l'interrégion Méditerranée.

Direction de l'ACR

Franck Braemer, Michel Pasqualini, Emmanuel Pellegrino

Organisation de la table ronde

Jean-Pierre Brun, directeur du Centre Jean Bérard

Franck Braemer et Didier Binder, directeurs du Cépam

Secrétatriat de la table ronde

Cépam : Jeannine François

Fréjus, Service du Patrimoine : Marina Dutartre

Centre Jean Bérard : Maria Francesca Buonaiuto, Antonietta Brangi, Anna Maria Gallo

\section{Édition}

Secrétariat d'édition

Fréjus, Service du Patrimoine : Pascale Denis et Michel Pasqualini

Centre Jean Bérard : Luca Basile, Maria Francesca Buonaiuto, Richard Bertaux

Infographie

Fréjus, service du Patrimoine : Joris Pâques

Centre Jean Bérard : Clément Pasqualini, Richard Bertaux, Marina Pierobon

Maquette

Centre Jean Bérard : Clément Pasqualini et Jean-Pierre Brun

Couverture

Marina Pierobon

Relecture des textes

Maria Francesca Buonaiuoto, Nuria Nin et Michel Pasqualini 


\section{Institutions associées}

\section{ITALIE}

Soprintendenza archeologica per le province di Napoli e Caserta Soprintendenza per i beni archeologici della Liguria Soprintendenza archeologica di Pompei

Facoltà di lettere e filosofia, Università La Sapienza, Roma

Università di Genova, Dipartimento per lo studio del territorio e delle sue risorse Università di Bologna - Facoltà di conservazione dei beni culturali - Sede di Ravenna

Università Federico II, Dipartimento scienze della terra, Napoli Università del Molise

Istituto internazionale di studi liguri

\section{FRANCE}

DRASSM, Marseille

Service régional d'archéologie de Provence-Alpes-Côte d'Azur

\section{UMR 6130, CÉPAM / CNRS-UNSA}

UMS 1797, Centre Jean Bérard, CNRS - École française de Rome

UMR 6573, Centre Camille Jullian, CNRS-Université de Provence

UMR 5138, Archéométrie et archéologie, Maison de l’Orient et de la Méditerranée, Lyon

UMR 5607, Ausonius CNRS - Université de Bordeaux III

Université Lumière-Lyon 2

Université d'Aix-Marseille 2

INRAP (Institut national de recherches archéologiques préventives)

Pôle archéologique départemental, Conseil Général du Var

Musée archéologique de Nice-Cimiez (Ville de Nice)

Musée archéologique intercommunal, Istres

Ville de Fréjus, Direction de la culture, Service du patrimoine

Ville d'Aix-en-Provence, Mission archéologie

Ville d'Arles, Service archéologique

Centre archéologique du Var, Toulon 
...Vous verrez, de poème en poème tout ce que l'on jette en chemin! On se déleste en avançant. Pourtant, tout est à recommencer, toujours :

l'atteinte du sommet est un leurre...

René Char, Dans l'atelier du poète Quarto Gallimard, Paris, 2003, p. 835 


\section{SOMMAIRE}

Action Collective de Recherche sur les céramiques communes de Marseille à Gênes

BiLAN ET PERSPECTIVES

Frank Braemer, Michel Pasqualini, Emmanuel Pellegrino

Les ateliers de potiers en Provence

INVENTAIRE RAISONNÉ

Pierre Excoffon

L'ATELIER DE POTIER ANTIQUE D'AMPHOUX

(Fox-AMphoux, VAR)

Jean-Marie Michel

UN ATELIER DE POTIERS DES II ${ }^{\mathrm{e}}$-III ${ }^{\mathrm{e}}$ SIÈCLES AP. J.-C.

Rue du Suveret, Fréjus (Var)

Emmanuel Botte et Pierre Excoffon

L'atelier de potier de Portissol

(SANARY-SUR-MER, VAR)

Henri Ribot, Didier Martina-Fieschi

La production de céramique commune à Pompéi

UN FOUR DE POTIER EN REGIO I, INSULA 5, 2

Laëtitia Cavassa

La production de céramique commune à Pompéi

STUdio PETROGRAFICO SUI REPERTI CERAMICI

Celestino Grifa, Vincenzo Morra

Rinvenimento di una fornace d’età bizantina a Cuma

L'area della « Cava Greca » a Cuma

Paolo Caputo, Cristina Regis

Scarti di Ceramica comune di età ellenistica dallo scavo di piazza Nicola Amore a Napoli : DATI PRELIMINARI SULLA PRODUZIONE

Stefania Febbraro, Daniela Giampaola



Anne Schmitt, Nadia Cantin, Valérie Thirion-Merle

Les céramiques communes de la Provence romaine
Fabrications locales et importations II ${ }^{\mathrm{e}}$ Siècle av. N. E. / III ${ }^{\mathrm{e}}$ Siècle de N. E.

Michel Pasqualini

La CÉramique Commune d'époque romaine dans le déPartement des Alpes-Maritimes 
LES PROductions de CÉRAMIQUe À PÂTE CALCAIRE ENTRE LA FIN DU

$I^{\text {er }}$ S. AV. N. È. ET LA FIN DU I ${ }^{\text {er }}$ S. DE N. È. À PARTIR DES ATELIERS

de Saint-Lambert / Valescure et de Sainte-Croix 2 (FréJus, Var)

Aurélie Dumont, Chérine Gébara

LES OFFICINES dE POTIERS de VELAUX (13) : LES PRODUCTIONS

Emilie Porcher

Les céramiques communes d'origine orientales dans le Sud de la Gaule au Haut-Empire

Le gobelet Marabini LXVIII

Emmanuel Pellegrino

Céramiques communes importées d’Italie en Provence II ${ }^{\mathrm{e}}$ siècle av. N. È. / III ${ }^{\mathrm{e}}$ Siècle de N. È.

Michel Pasqualini, Antoine Pasqualini, Clément Pasqualini

Ceramica non tornita ad imitazione di Produzioni massaliote dallo scavo di Corti

CONTESTO, DIFFUSIONE E PRODUZIONE

Francesca Chiocci, Claudio Capelli

Le Ceramiche comuni di Cuma

Alberto De Bonis, Laetitia Cavassa, Celestino Grifa, Alessio Langella, Vincenzo Morra

Produzione e commercio di alcune classi di ceramica comune

Nel territorio di Vado Ligure (SV) (I - III SEC. D.C.)

Primi dati archeologici ed archeometrici integrati : Part. 1

Francesca Bulgarelli, Eleonora Torre

Produzione e commercio di alcune classi di ceramica comune

NEL TERritorio di VAdo Ligure (SV) (I - III SEC. D.C.)

PRIMI DATI ARCHEOLOGICI ED ARCHEOMETRICI INTEGRATI

Part. 2 : Analisi in microscopia ottica

Claudio Capelli, Roberto Cabella

Classification des céramiques communes provençales romaines.

Productions des bassins d’Arles et du Rhône, de l'Arc (Aix-en-Provence), de l'Huveaune (Marseille), de l'Argens (FréJus) et de la Siagne (Cannes/Mandelieu) I $^{\text {er }}$ S. AV. N. È. - III' ${ }^{\mathrm{e}}$ S. DE N. È.

Michel Pasqualini

TYPOLOGIE DIACHRONIQUe ET DIFFUSION DE LA CÉRAMiQue MODELÉE DU VAR DU II' ${ }^{\mathrm{e}}$ s. AV. J.-C. AU III ${ }^{\mathrm{e}}$ s. AP. J.-C.

Jacques Bérato

Gobelets À ANSE À POUCIER EN CÉRAMIQUe NON TOURNÉE DE MÉDITERRANÉE NORD-OCCIDENTALE

Frédéric Marty, Claudio Capelli et Roberto Cabella

La fornace « B » di Alcamo Marina : produzione della forma Dressel 21-22 (Trapani- Sicilia)

Xabier González Muro

Corinne Sanchez 
La vaisselle en usage à Aix-en-Provence

ENTRE LE I ${ }^{\mathrm{er}}$ SIÈCLE AV. J.-C. ET LES II $/$ IIII $^{\mathrm{e}}$ SIÈCLES AP. J.-C.

Núria Nin, Mélissa Savanier

Le mobilier issu des fouilles de la villa Régine au Le Puy-Sainte-Réparade

Philippe Chapon et Michel Pasqualini

Les Céramiques Communes des gisements du RhôNe à ArLes

LE FACIÈS PORTUAIRE D'ÉPOQUE IMPÉRIALE

Luc Long, Jean Piton, David Djaoui

Fosse d'eXTRAction d'ARgILE ET DÉPOTOIR DE POTIER DES II'/IV ${ }^{\mathrm{e}}$ SIÈcLES DE N. È. aux Paluns et à la Pinède (La Cadière et Le Castellet, Var)

Pascal Alliot, Jean-Michel Théveny

La céramique commune de l'espace sud des thermes de l'Est de Cemenelum

à Cimiez (Nice, Alpes-Maritimes)Un contexte du Haut-Empire et de l'Antiquité tardive

Alain Grandieux

Ceramiche tardo antiche da Pompei

Ernesto De Carolis, Celestino Grifa, Alessio Langella, Vincenzo Morra, Gianluca Soricelli

LA CERAMICA COMUNE E DA CUCINA

DA CONTESTI TARDO ANTICHI dA NAPOLI

Vittoria Carsana

Bibliographie gÉNÉRALE

LES AUTEURS 


\title{
La production de céramique commune à Pompéi Un four de potier dans l'insula 5 de la regio I
}

\author{
Laëtitia Cavassa*
}

Depuis 2001, l'artisanat constitue le thème principal des recherches menées par le Centre Jean Bérard à Pompéi. De nombreuses activités ont été étudiées sur l'ensemble du site, depuis la parfumerie jusqu'à la teinture des tissus ou la tannerie (Borgard et al., 2005). Il reste toutefois une branche artisanale encore assez mal connue : celle de la production céramique. Les conditions d'ensevelissement et de conservation de ce site font de la découverte d'un four de potier et de sa production un élément important pour la connaissance de cet artisanat dans la cité antique. À l'heure actuelle, très peu de fours ont été reconnus à Pompéi. Nous en connaissons un en Reg. I, ins. 20, 3 ayant produit des lampes à huile durant le $\mathrm{I}^{\mathrm{er}}$ siècle de notre ère (Annecchino 1977). Les fouilles du XIX ${ }^{\mathrm{e}}$ siècle ont également révélé des fours artisanaux ayant certainement produit de la céramique, le long de la via dei sepolcri (Breton 1855,



Fig. 1 - Plan de Pompéi. La tannerie se trouve en Reg. I, ins. 5

* Ingénieur CNRS - Centre Jean Bérard 


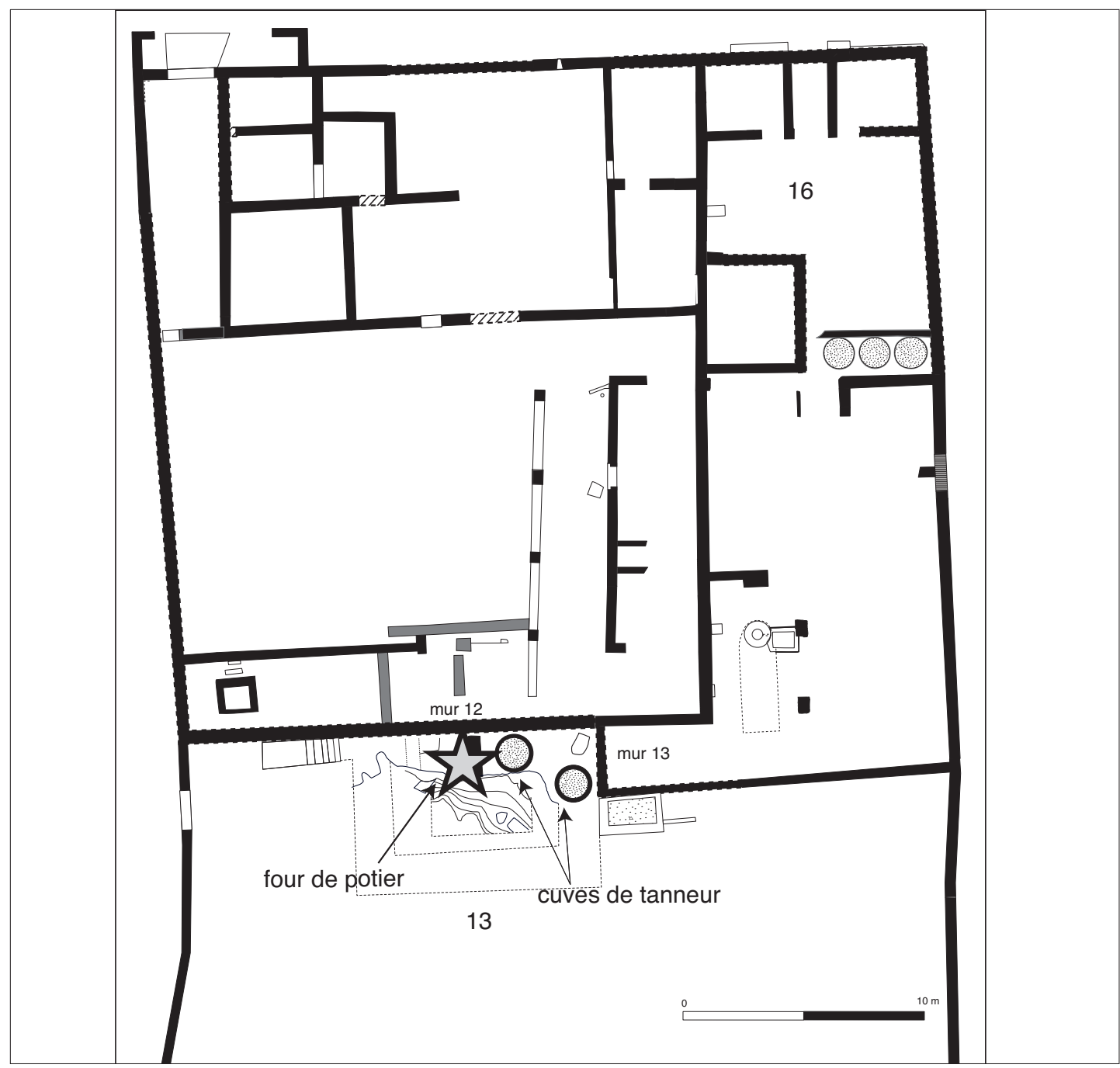

Fig. 2 - Plan général de la tannerie.

Fiorelli 1875, et Annecchino 1977). Mais quelle était la typologie des céramiques produites dans ces fours ? Cette information fait défaut et constitue le point essentiel de toute recherche concernant les fours de potiers à Pompéi. Les récentes fouilles du Centre Jean Bérard menées dans la tannerie donnent quelques précisions concernant l'ars vasaria.

\section{La tannerie en Reg. I, ins. 5, 2}

La tannerie de Pompéi se trouve dans la partie sud de la ville, à proximité de la porte de Stabies (fig. 1). Au moment de l'éruption du Vésuve, cet établissement artisanal occupe tout l'îlot 5 de la région I. L'édifice, dégagé en 1873-1874, fait depuis 2001 l'objet de recherches approfondies qui concernent son histoire et ses fonctionnalités (Leguilloux 2004, p. 44-50, Borgard et al. 2005, p. 197-199, Botte et al. 2006, Leguilloux 2007)1 ${ }^{1}$. Lors de la campagne de fouille de 2005, les recherches se sont concentrées sur le secteur sud de l'îlot, traditionnellement identifié comme un jardin, mais qui s'est avéré être au moment de l'éruption une vaste carrière de lave partiellement comblée de détritus ${ }^{2}$. Nous y avons mis au jour un four de potier ayant fonctionné avant l'installation

1 Les fouilles sont menées sous la direction de Martine Leguilloux que nous remercions de nous avoir confié l'étude et la publication de ce four.

2 Chaque pièce de l'édifice a été numérotée. La zone du "jardin" où a été mis au jour le four porte le numéro 13. Chaque pièce ou élément mentionné dans l'article sera nommé par son numéro. 
de la tannerie (fig. 2). Un bref rappel chronologique de l'évolution de cet îlot est nécessaire avant d'en venir à la description du four. Les recherches stratigraphiques ont permis d'établir que la tannerie a été installée sur d'anciennes demeures, dont la plus ancienne remonte à la première moitié du $\mathrm{III}^{\mathrm{e}}$ siècle avant notre ère. La tannerie a connu deux grandes phases dont une antérieure au tremblement de terre de 62 ; ce sont des cuves de cette première phase qui ont recoupé le four de potier.

\section{Le four}

Le four a été mis au jour au nord del'espace 13 mais il n'en reste que très peu d'éléments : un morceau de paroi large d'une cinquantaine de centimètres et conservé sur environ 1,80 m de long. Ce mur (MR 148) est constitué de blocs de basalte et de tuf pour le parement extérieur, et de fragments de briques liées à l'argile pour le parement interne. Un fragment de la chambre de chauffe formée de tegulae est également conservé. Les traces de rubéfaction nous permettent de supposer qu'il s'agit d'une paroi $\mathrm{du}$ laboratoire du four (fig. 3a et b). Nous nous limiterons dans ce présent article à présenter les phases d'occupation en relation directe avec l'histoire du four et susceptibles d'apporter des éléments de chronologie (fig. $4 a$ et b).

\subsection{LA MISE EN PLACE DU FOUR}

La première phase d'occupation est caractérisée par une série de sols datables des $\mathrm{II}^{\mathrm{e}}$ et $\mathrm{I}^{\text {er }}$ siècles avant J.-C. qui reposent sur le substrat et qui sont recoupés par la construction du four de potier dont la fondation atteint le substrat volcanique. Comme nous l'avons vu, le seul élément conservé du four est son mur ouest. Il est construit dans un remblai (US 13-037) qui comporte deux fragments de céramique sigillée italique. Il s'agit d'un plat de forme Ettlinger 4 et d'une coupe de forme Ettlinger 14.2. À partir de ces deux fragments, nous pouvons établir un terminus post quem pour l'installation du four, à savoir après le début de notre ère, peut-être vers la fin de la période augustéenne ou sous le règne de Tibère (Ettlinger et al.1990, p. 58-59 et 76-77).

\subsection{LE FONCTIONNEMENT DU FOUR}

La période d'utilisation du four constitue la deuxième phase. De celle-ci, nous ne conservons que peu d'éléments : un fragment de la chambre de chauffe et la dernière couche d'utilisation du

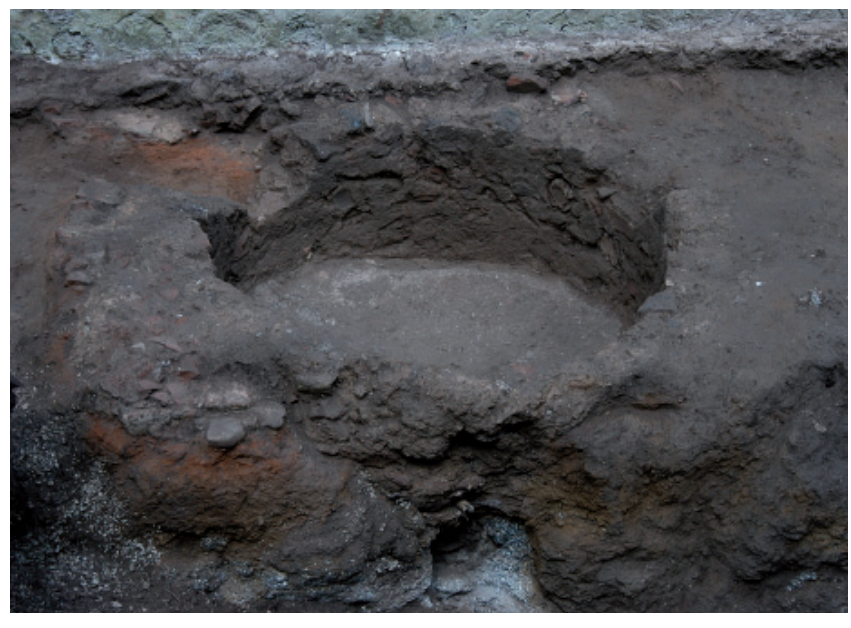

Fig. 3a - Vue du mur ouest du four (M148) et de la cuve 16

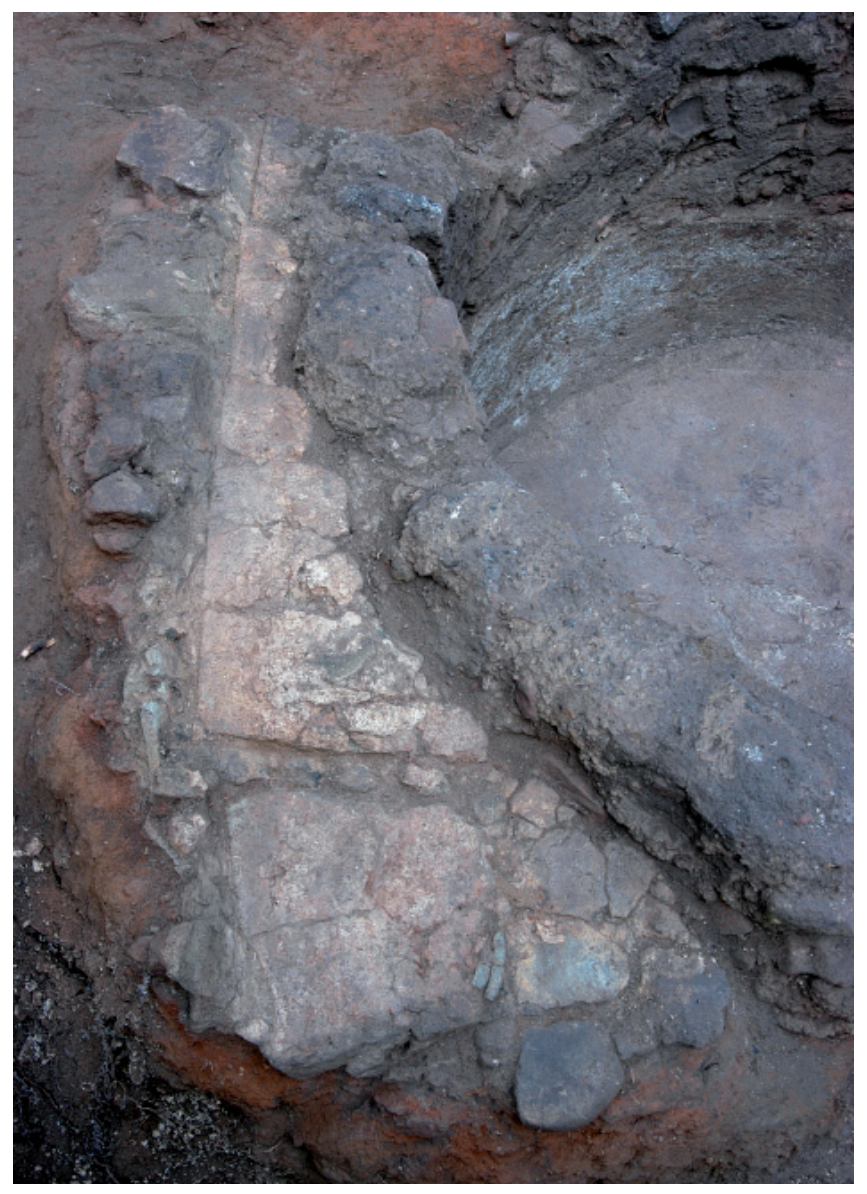

Fig. 3b - Détail du laboratoire du four en partie détruit par l'installation de la cuve 16 


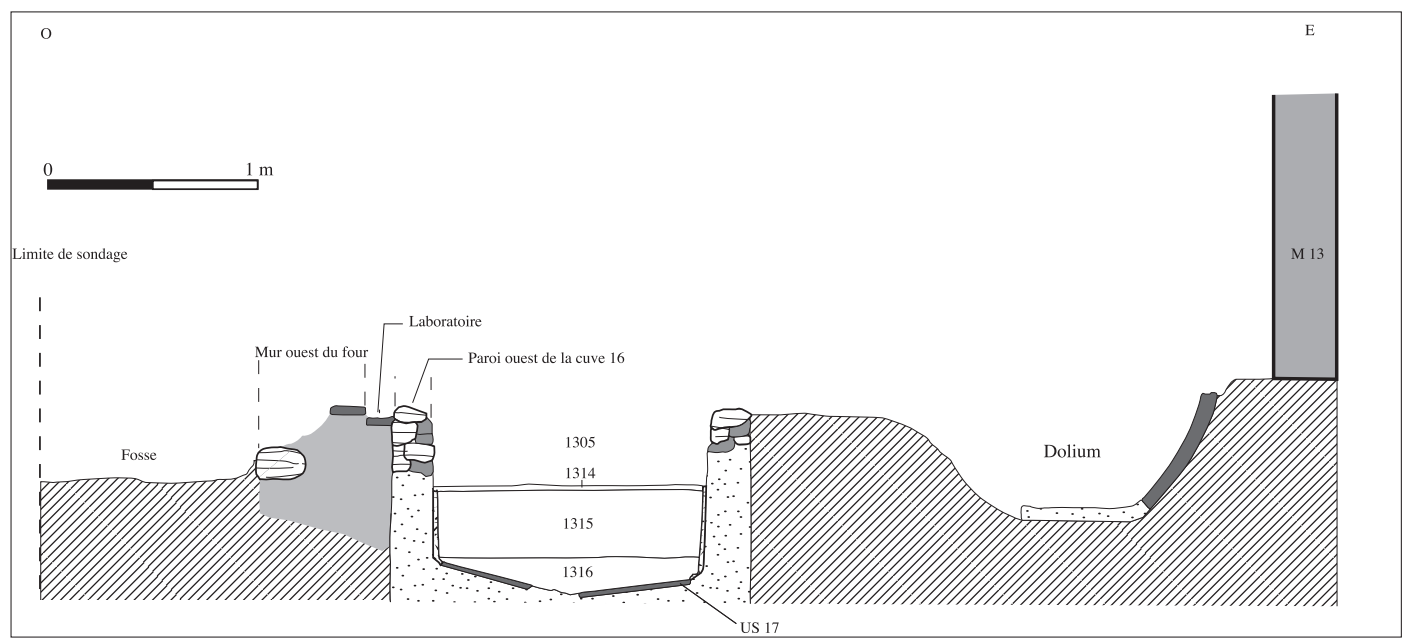

Fig. 4a - Stratigraphique du secteur 13 : coupe ouest-est du four et de la cuve 16

four (US 13-023). Cette strate est constituée de cendre et argile rubéfiées. Très peu de matériel a été mis au jour dans ce niveau en partie détruit. Nous avons recensé 134 fragments de céramique représentant 19 vases au minimum. La céramique commune italique, représentée par 132 fragments, soit $98 \%$ du matériel mis au jour, est composée de treize ollae de forme Di Giovanni 2311a (fig. 5, LCP1) (Di Giovanni 1996, p. 92, fig. 19), de six couvercles dont un est surcuit (fig. 5, LCP3), d'une urne et de nombreuses panses de forme non identifiable.

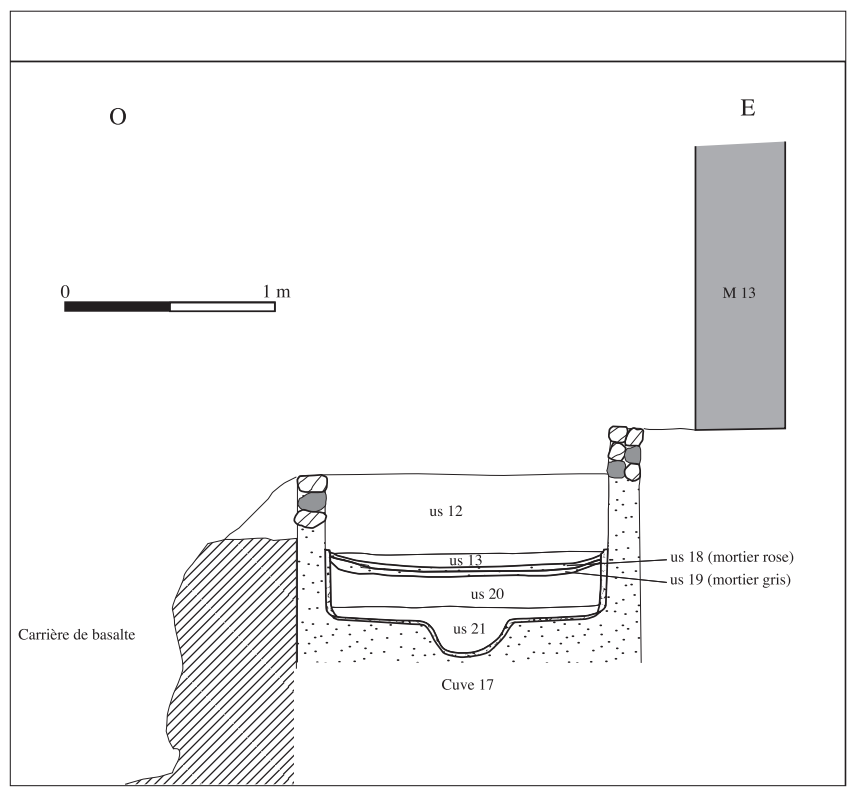

Fig. 4b - Stratigraphique du secteur 13 : coupe ouest-est de la cuve 17
Le reste du matériel, soit $2 \%$, se compose de deux fragments de céramique sigillée orientale A (ESA). Il s'agit d'une coupelle peu profonde avec un bord à marli. Son diamètre est de $17,5 \mathrm{~cm}$. La forme n'est pas répertoriée dans la typologie de J.W. Hayes (voir Atlante 1985). Cette catégorie de vases ayant été produite pendant près de quatre siècles $\left(\mathrm{II}^{\mathrm{e}} \mathrm{s}\right.$. av. J.-C.-II ${ }^{\mathrm{e}}$ siècle ap. J.-C.), sa présence est insuffisante pour cerner la chronologie.

À l'est du four, au pied du mur 13, on retrouve une couche de terre cendreuse (US 13-024), dans laquelle a été installée, lors de la phase suivante, une cuve de tanneur (cuve 17). Cette couche correspond à la période de fonctionnement du four. 150 fragments céramiques en proviennent et correspondent à 23 vases. La céramique commune représente $83 \%$ du total. Comme dans le dernier niveau du four, on y trouve six bords d'ollae de type Di Giovanni 2311a, dont un est surcuit, des couvercles et des fragments de panses non déterminables. Parmi le reste du matériel, signalons une panse de céramique campanienne $\mathrm{A}$, un fragment de gobelet à paroi fine de type Mayet 2 dont la production est attestée du dernier quart du $\mathrm{II}^{\mathrm{e}} \mathrm{s}$. au premier quart du $\mathrm{I}^{\mathrm{er}}$ siècle av. J.-C (Mayet 1975, p. 27). Les attestations les plus récentes de ce type de gobelet sont datées des environs du milieu du I $^{\text {er }}$ siècle av. J.-C (Gervasini 2005, p. 296). Enfin, nous dénombrons quelques fragments d'amphores italiques produites dans l'aire vésuvienne. Nous avons également mis au jour dans ce niveau six objets circulaires en céramique, d'une dizaine de centimètres de diamètre et d'une épaisseur de 2-3 centimètres. Il s'agit de séparateurs utilisés dans les fours et placés entre chaque vase 
pour éviter qu'ils ne se soudent l'un à l'autre (fig. 6a, b et c). Le four, ces séparateurs, le couvercle et le bord d'olla de forme Di Giovanni 2311a sont autant d'éléments confirmant l'activité d'un potier. Dans l'état actuel de nos connaissances, il semble que seule de la céramique culinaire ait été produite.

\subsection{LA DESTRUCTION ET L'ABANDON DU FOUR}

La troisième phase correspond à l'abandon et la destruction du four certainement entraînés par l'installation des deux cuves de tanneur (cuves 16 et 17) et d'un dolium. La cuve 16 est construite sur le four. Son creusement cause donc la destruction quasi intégrale de la structure. La cuve 17, construite à l'est du four, est installée sur la couche cendreuse (US 13-024) correspondant aux déchets de la dernière phase d'utilisation du four. Les cuves sont caractérisées par la présence de deux pavements superposés témoignant de deux états successifs de fonctionnement. Entre chaque sol, un peu de matériel a été mis au jour. Celui-ci donne des indications chronologiques supplémentaires concernant le fonctionnement des cuves et la destruction du four. Le niveau le plus ancien de la cuve 16, l'US 13-017, n'a pas livré de mobilier. Il est recouvert par deux couches (US 13-016 et 15) sur lesquelles sera mis en place le second sol. Ce remblai assez homogène a livré quelques fragments de céramique sigillée italique, un bol - archéologiquement complet- à paroi fine de forme Mayet XXXIII, dont la production est attestée durant le $1^{\text {er }}$ quart du $1^{\text {er }}$ siècle de notre ère (Mayet 1975, p. 67), de la céramique commune, un bord d'amphore vinaire italique de production locale de forme Dressel 2-4. Les données fournies par le matériel semblent indiquer que ce deuxième sol a été mis en place durant la I ${ }^{\text {ere }}$ moitié du ${ }^{\text {er }}$ siècle de notre ère. La cuve 17 a également subi une réfection. Entre les deux sols (US 13-018-19 et 13-022), un niveau de remblai (US 13-020) a été identifié : il contient de la céramique commune italique, quelques fragments de vases à paroi fine et de deux assiettes en céramique sigillée italique, forme Ettlinger 10.1.2 et 12, datées du milieu et de la fin de la période augustéenne, donc résiduelles (Ettlinger et al. 1990, p. 68-69 et p. 72-73).

Compte-tenu de ces éléments, il paraît vraisemblable que le deuxième sol des cuves a été mis en place à partir du $2^{\mathrm{e}}$ quart ou vers le milieu du $\mathrm{I}^{\mathrm{er}}$ siècle de notre ère. On peut donc en déduire, avec précaution, que la première phase de construction des cuves se situe quelques années auparavant. Le four a donc dû fonctionner jusqu'à cette date.

\subsection{LA PRODUCTION}

Le matériel mis au jour dans les niveaux correspondant à la dernière phase d'utilisation du four (US 13-023 et 24) est peu abondant. Toutefois, ont été identifiés plusieurs fragments d'ollae en céramique commune de type Di Giovanni 2311a, parmi lesquels un bord surcuit (fig. 5, LCP2). Associé à ces fragments d'ollae, un bord de couvercle de petit diamètre, en céramique commune, est également surcuit. Enfin, l'activité artisanale est confirmée par six fragments de séparateurs circulaires. 19 autres fragments de séparateurs (US 13-001 et 02), représentant 10 objets, ainsi que 2 séparateurs en patte de coq (fig. 5, LCP4, et fig. 6a, b et c) ont été mis au jour dans des niveaux de surface (US 13-006). Les séparateurs n'apportent pas de renseignements concernant la chronologie. Toutefois la présence de deux types différents, circulaires et en patte de coq, nous incite à penser que le four a produit d'autres formes de vases. En effet, les séparateurs circulaires, selon une reconstitution effectuée à partir du matériel de métaponte, serviraient surtout à empiler des vases de forme fermée, tels que les ollae. Le séparateur était posé sur la gorge interne du bord ${ }^{3}$ d'un premier vase, puis en recevait un second (Cracolici 2003, p. 51-56). Ce procédé permettait d'empiler les vases en évitant qu'ils ne restent collés les uns aux autres. Le deuxième type, en patte de coq, serait plutôt employé pour la cuisson des vases de forme ouverte. Des exemplaires identiques ont été mis au jour dans un atelier ayant fonctionné durant la période augustéenne à Bram dans l'Aude, où les assiettes en céramique sigillée portent les traces des trois branches des séparateurs (Passelac 2001, p. 152, fig. 10). M. Passelac y verrait un des aspects du savoir-faire italique importé en Gaule. Les séparateurs, posés au centre des plats, seraient utilisés pour éviter l'affaissement des vases après le tournage et pour les séparer les uns des autres au moment de la cuisson (Passelac 1996a et b, p. 14-15).

L'ensemble de ces éléments indique que le four a produit, parmi d'autres formes, des pots de forme Di Giovanni 2311a, comme le laisse supposer la présence d'un bord surcuit de ce type d'olla (fig. 7). Il s'agit d'un pot pour la cuisson des aliments, à fond plat, sans anse. La lèvre est oblique, en forme d'amande. Cette forme est attestée à Pompéi dans des contextes du ${ }^{\text {er }}$ siècle avant J.-C. jusqu'au niveau de 79 de notre

3 Le séparateur est posé à l'endroit où le diamètre de l'encolure est le plus étroit. 


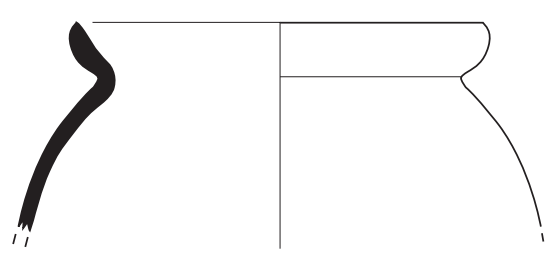

Us 13.23

LCP 1

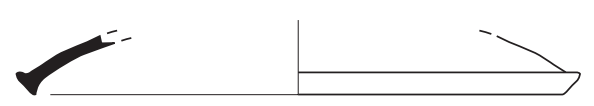

Us 13.23

LCP 3

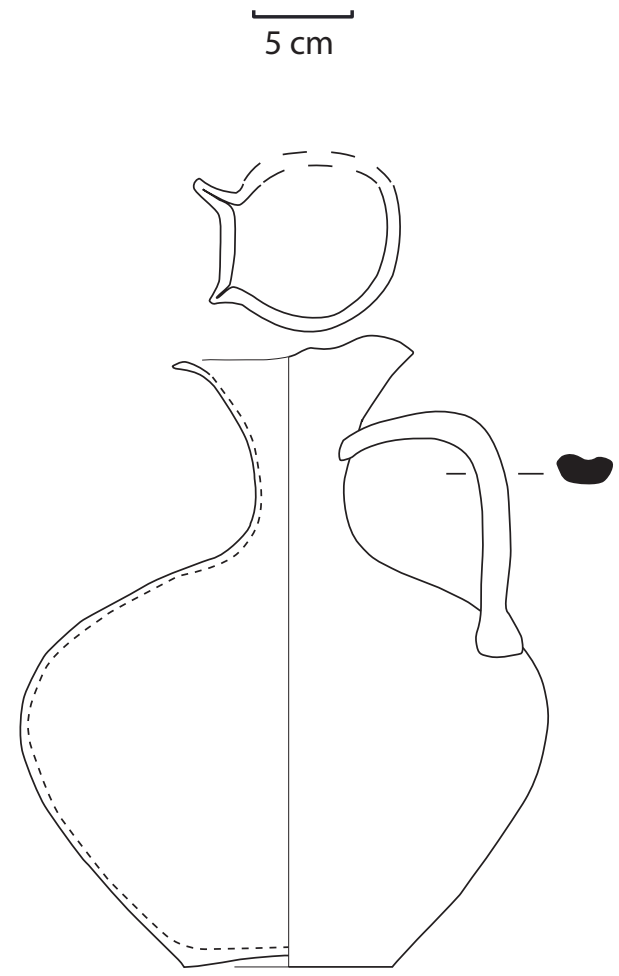

Us 13.13

LCP 5

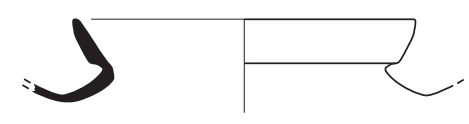

Us 13.24

LCP 2

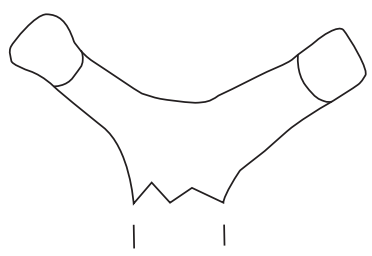

Us 13.06

LCP 4
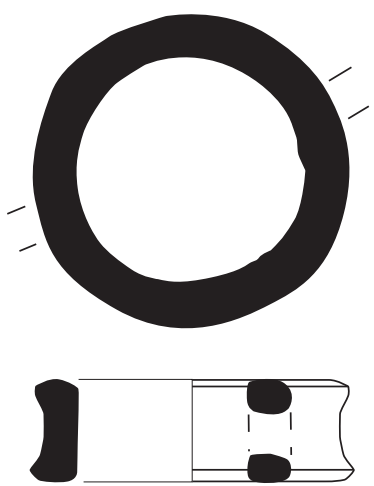

Us 13.00

LCP 6
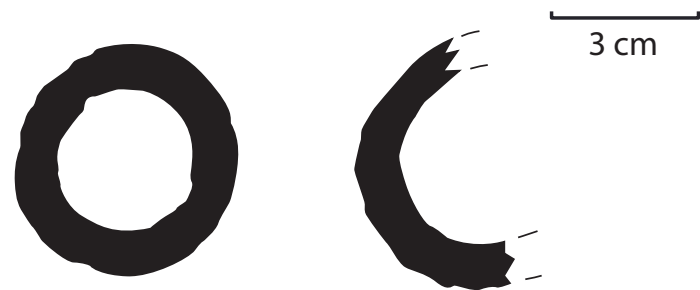

O

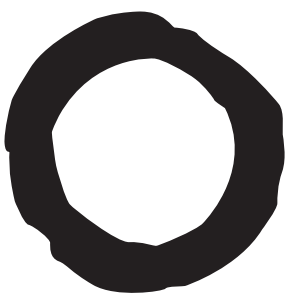

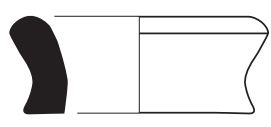

Us 13.00

LCP 7
Us 13.00

LCP 8

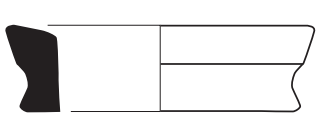

Us 13.24

LCP 9

Fig. 5 - Échantillons analysés. Les échantillons LCP1, LCP2, LCP3, et LCP5 sont réduits au 1:3, les séparateurs (LCP4 et LCP6 à 9) sont réduits au 1:2 

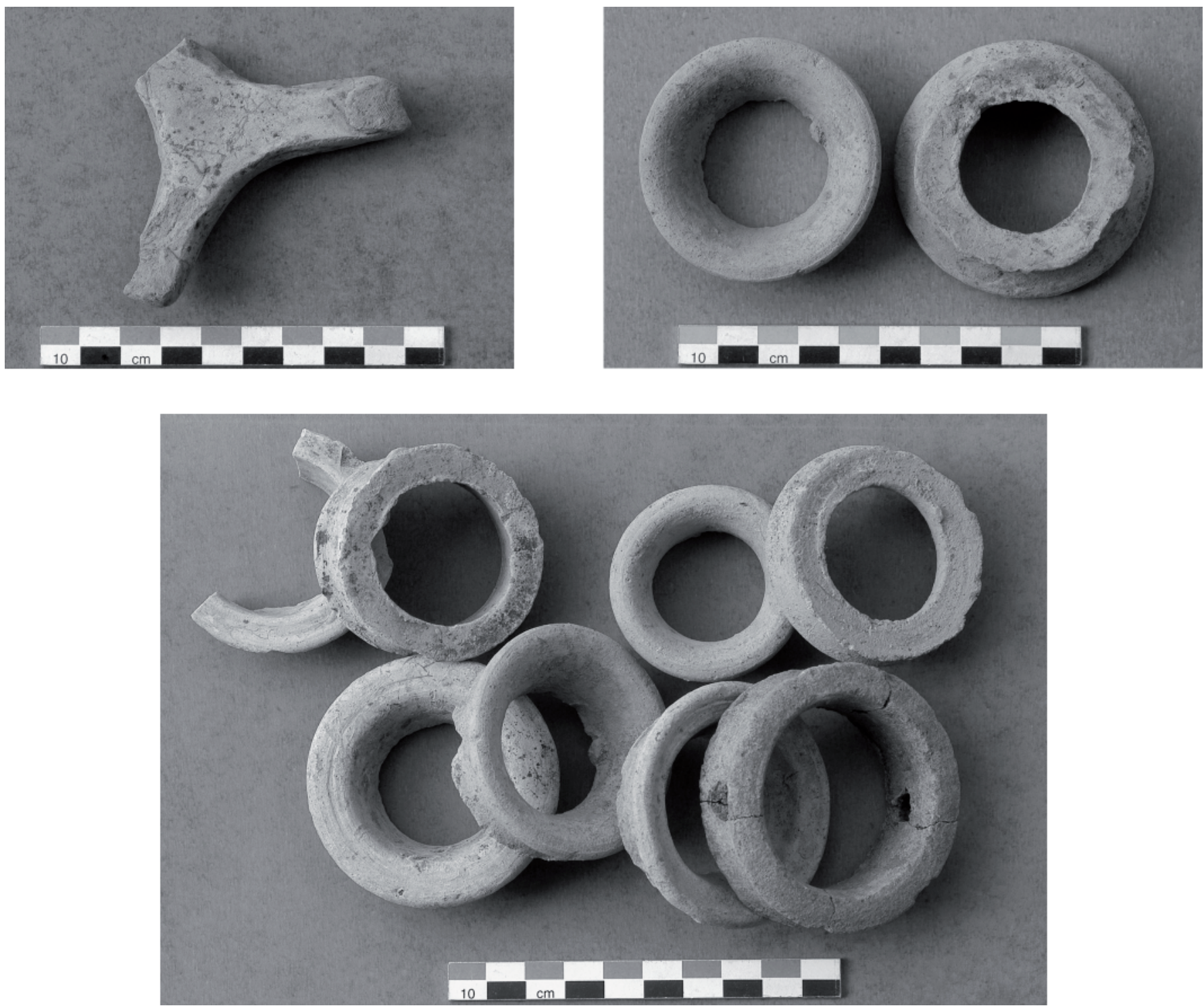

Fig. 6a, b et c - Séparateurs circulaires et en patte de coq
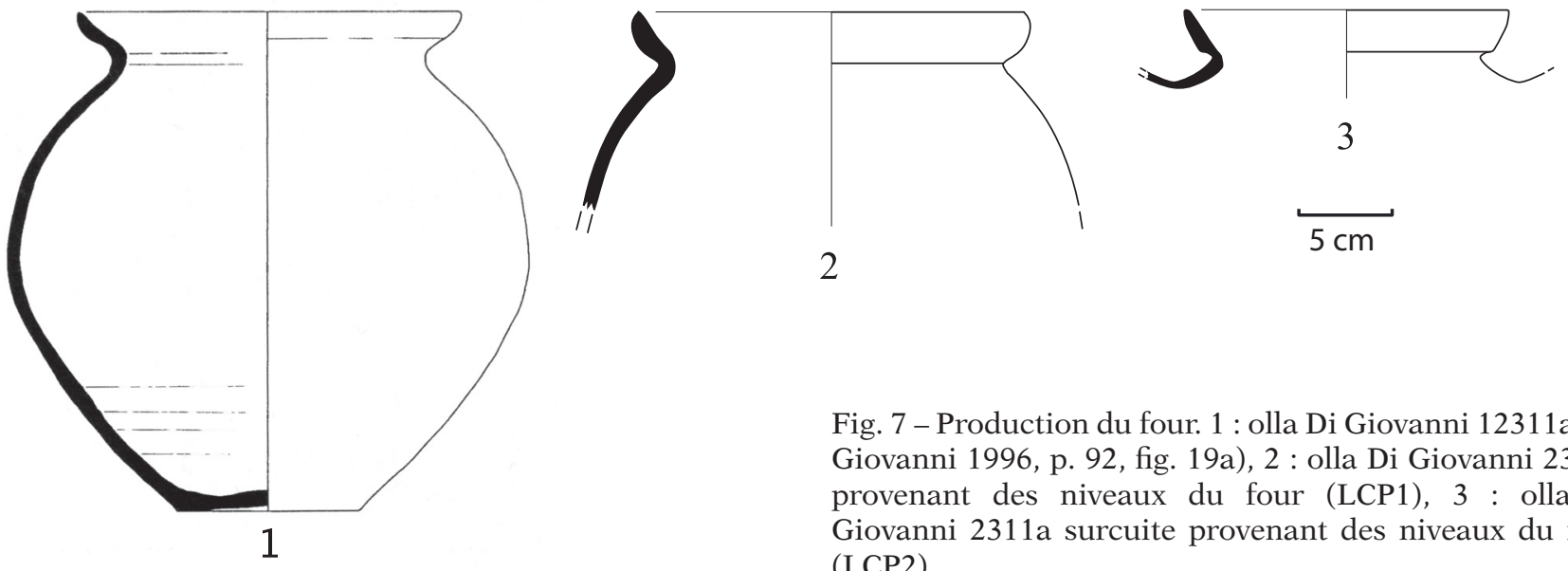

Fig. 7 - Production du four. 1 : olla Di Giovanni 12311a (Di Giovanni 1996, p. 92, fig. 19a), 2 : olla Di Giovanni 2311a provenant des niveaux du four (LCP1), 3 : olla Di Giovanni 2311a surcuite provenant des niveaux du four (LCP2) 
ère, et en Italie de la fin de la République au $\mathrm{II}^{\mathrm{e}}$ siècle de notre ère (Di Giovanni 1996, p. 92). Vincenzo Di Giovanni, se basant sur les rapports diamètre / hauteur des vases, a réparti les 133 exemplaires conservés dans le dépôt des Granai, en trois groupes de mesures.

Les " petits vases » (diamètre d'ouverture : 12 à13 cm, hauteur 17 à $18 \mathrm{~cm}$ ), les vases de taille moyenne (diamètre d'ouverture 15 à $16 \mathrm{~cm}$, hauteur : 22 à $24 \mathrm{~cm}$ ) et les grands vases (diamètre d'ouverture : 17 à $18 \mathrm{~cm}$, hauteur : 26 à $28 \mathrm{~cm}$ ). Notre échantillon (LCP1) appartient au premier groupe des petits vases, avec un diamètre de 12,4 cm. Ce groupe est rangé par V. Di Giovanni dans la catégorie des productions campaniennes. Le fragment surcuit (LCP2) a un diamètre d'ouverture légèrement plus petit $(10,4 \mathrm{~cm})$, peut-être surcuit.

Cette étude concernant ce four et sa production d'ollae s'accompagne d'analyses chimiques réalisées par le laboratoire du département des Sciences de la Terre de l'Université Federico II de Naples. Parmi le mobilier, nous avons sélectionné onze échantillons (fig. 5) provenant des niveaux de fonctionnement du four, de la cuve 17, du dépotoir de la tannerie constitué dans la fosse d'extraction et enfin des niveaux de surface ${ }^{4}$.

Provenant du niveau de cendres (US 13-023) reposant sur la sole du four, ont été analysés : un fragment d'olla de forme Di Giovanni 2311a (LCP1), un fragment de couvercle surcuit en céramique commune (LCP3), deux éléments rubéfiés provenant de la paroi du four (LCP10 et 11), le fragment surcuit d'olla de forme Di Giovanni 2311a (LCP2), ainsi qu'un séparateur circulaire (LCP9) mis au jour dans la couche de cendres (US 13-024) correspondant au niveau de fonctionnement du four. Parmi les séparateurs circulaires, plusieurs se distinguent par leur pâte, et pour cette raison trois autres, provenant cette fois de la couche de surface (US 13-001), ont été analysés (LCP 6, 7 et 8) ainsi qu'un fragment de séparateur en patte de coq (LCP4) provenant d'un niveau de dépotoir de la tannerie (US 13-006). Enfin, une cruche (LCP5) en céramique commune de type Gasperetti 1362a a été analysée (Gasperetti 1996, p. 47). Elle provient d'une fine couche de terre (US 13-013) reposant sur le dernier sol (US 13-018) de la cuve 17.

4 Chaque échantillon est nommé par les initiales LCP, suivies du numéro allant donc de 1 à 11 .

\section{Les fours de potiers et la production céramique à Pompéi}

Les recherches conduites sur le four mis au jour dans le secteur sud de la tannerie offrent l'occasion d'une mise au point sur la production céramique à Pompéi. Plusieurs sources sont à notre disposition (fig. 8).

\subsection{LES FOURS}

À ce jour, six fours ont été identifiés, mais très peu ont fourni un témoignage précis concernant leur production. Un premier four a été mis au jour durant la $1^{\text {ère }}$ moitié du XIX ${ }^{\mathrm{e}}$ siècle au nord de la ville, le long de la via dei sepolcri, au n. 29 (Fiorelli 1875, p. 416). Ernest Breton qui a transmis ses impressions de voyage sous forme d'un guide de visite du site présente cette "fabrique de poteries » dont le four est "d'une construction très remarquable » (Breton 1855, p. 234-35). La production n'est toutefois mentionnée nulle part. Dans le même secteur, un autre four est découvert en 1875. Les journaux de fouille mentionnent la découverte de 34 " marmites " près du four, sans autre précision (Annecchino 1977, p. 106). Un troisième établissement, certainement le mieux documenté, a été dégagé en octobre 1958 par A. Maiuri, au sud de la voie de l'Abondance en Reg. I, ins. 20, 3. L'ensemble de l'atelier était composé d'une partie destinée à la production et d'un espace de vente, le tout dans un bon état de conservation. La production de l'atelier a été retrouvée dans l'un des deux fours : 61 lampes et 123 petits vases appelés frittili. Avec les lampes, les fouilleurs ont mis au jour 24 moules (Cerulli Irelli 1977, p. 55-57). Lors des récents travaux menés par F. Coarelli et F. Pesando, dans la Regio VI, un four ayant produit des tuiles, des coupes et de grands conteneurs a été identifié dans des niveaux datés du $\mathrm{III}^{\mathrm{e}}$ siècle. Cette structure a été découverte sous les sols de la maison des Fleurs (Reg. VI, ins. 5, 8) dont la construction remonte au $\mathrm{II}^{\mathrm{e}}$ siècle avant J.-C (Coarelli, Pesando 2005, p. 169) . Dans l'insula 15 de la région VII, les mêmes chercheurs ont découvert un autre four ayant produit des amphores et de la céramique à vernis noir. Ce four a été détruit durant la seconde moitié du $\mathrm{II}^{\mathrm{e}}$ siècle avant notre ère par la construction de la maison Reg. VII, ins.15, 9 (Coarelli, Pesando 2005, p. 170). Le dernier four recensé est celui mis au jour dans la tannerie.

\footnotetext{
5 Concernant les coupes, les auteurs ne précisent pas de quelle production il s'agit.
} 


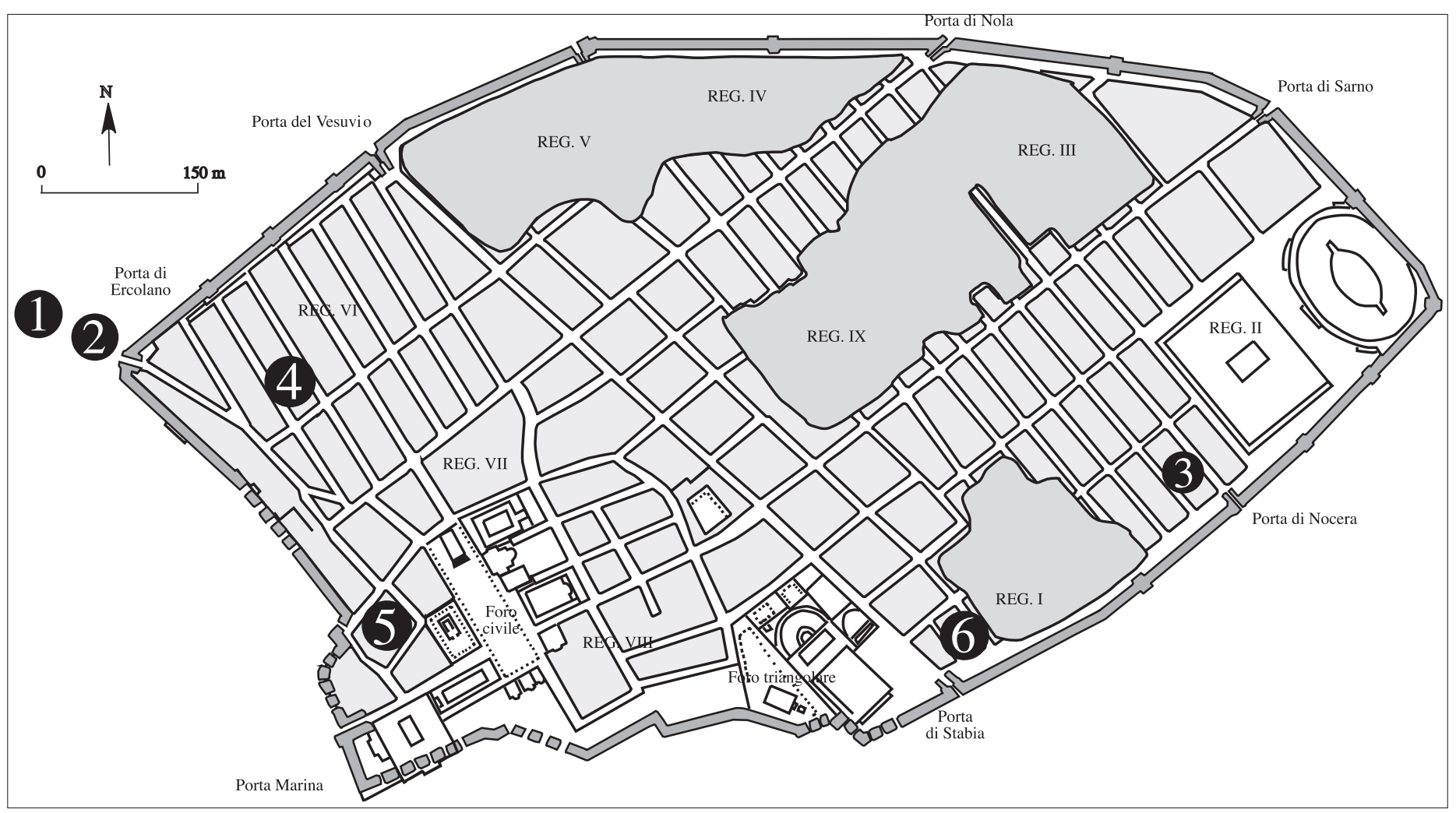

Fig. 8 - Plan général de Pompéi avec répartition des fours de potiers : 1 et 2 : via dei sepolcri, n. 29 ; 3 : Reg. I, ins. 20,3 ; 4 : Reg. VI, ins. 5,9; 5 : Reg. VII, ins. 15,9;6: Reg. I, ins. 5,2

\subsection{L'ÉPIGRAPHIE ET L'ICONOGRAPHIE}

Deux autres éléments pour la connaissance de l'artisanat à Pompéi, souvent pris en considération, sont l'iconographie et, dans une plus large mesure, l'épigraphie. Nombre de métiers sont en effet attestés par les enseignes de boutiques et les affiches électorales présentes sur les murs de la ville.

Une inscription retrouvée sur les murs de la caupona des praedia de Iulia Felix mentionne un "fabricant de jarres " (CIL IV, 10150).

CIL IV, 10150 : «Tu es tombé huit fois, mais tu serais bien capable de tomber seize fois. Tu as fait l'aubergiste, tu as eu un magasin de vases, tu as fait le charcutier, puis le boulanger et ensuite l'agriculteur, enfin tu t'es lancé dans le commerce des objets de bronze et tu as même fait le brocanteur ; maintenant tu te consacres à la fabrication des jarres... ».

Une autre inscription, gravée sur l'un des murs d'une taberna le long de la voie de l'Abondance en Reg. III, ins. 4, 1, dégagée en 1916 (Eschebach 1993, p. 104), donne l'index nundinarius, les jours de marchés (CIL IV, 8863). Cette inscription témoigne de l'itinéraire hebdomadaire d'un commerçant : lundi, Cumes ; mardi, Pouzzoles ; mercredi, Rome ; jeudi, Capoue ; vendredi, repos ? ; samedi, Pompéi, et dimanche, Nola.

Deuxième élément à prendre en compte : les " enseignes de boutiques ». La première se trouve en façade de l'édifice en Reg. I, ins. 8, 10, la taberna vasaria dite de Pulcinella. Cette structure a été dégagée en mars 1940 et a subi de nombreuses dégradations causées par les bombardements de 1943. La peinture se trouvait en fait sous une peinture plus récente trop abîmée et dont l'enduit s'est détaché de la paroi. Mesurant 1,30 $\mathrm{m}$ de long pour 0,57 $\mathrm{m}$ de hauteur, elle est composée de deux scénettes indépendantes. Il semble même que ces deux " tableaux » aient été réalisés par deux mains distinctes et à deux moments différents. La scène nous intéressant représente des potiers au travail. Au moins quatre artisans sont assis, occupés à tourner leur vase. Un cinquième personnage, une femme, se tient debout devant l'un des potiers, tenant deux vases dans ses mains. Cette enseigne correspondrait, selon A. Maiuri, non pas à un atelier mais à une boutique. Ce sont le manque d'espace et de structures caractéristiques qui l'ont fait pencher pour cette interprétation. Quoi 
qu'il en soit, si atelier ou boutique il y a eu, cela n'a pu fonctionner qu'avant le tremblement de terre de 62 ap. J.-C (Maiuri 1954, p. 90).

La deuxième enseigne a été mise au jour en façade d'un bâtiment se trouvant en Reg. II, ins. 3, 7-9. Elle se trouve sur la façade sud-ouest du bâtiment, et représente le dieu Vulcain debout, près d'un potier assis en train de tourner un vase. Près des pieds de l'artisan se trouvent cinq vases achevés (Della Corte 1939). A côté de la peinture se trouvent deux inscriptions peintes (CIL IV 7573 et 7574), dont l'une d'elles semble être une interdiction d'entrer (CIL IV, 7574).

\subsection{Les travauX de H. Eschebach}

H. Eschebach ne mentionne pas moins de cinq officinae vasariae et neuf läden vasarius, autrement dit neuf boutiques dont une ayant comme enseigne des vases peints (Eschebach 1993) .

Les cinq ateliers qu'il mentionne sont les suivants :

En Reg. I, ins.7, 4, l'interprétation repose sur la découverte d'un vase à garum sur lequel se trouvait l'inscription suivante : "P. Corneli / Corinti servos I fecit, qu'on peut traduire ainsi malgré les fautes : "Corinthus, esclave de Publius Cornelius, a fait ce vase »(Eschebach 1993, p. 38-39; CIL IV, 9683). Autrement dit nous aurions un urceus fabriqué par un certain Corinthus. Cette inscription sur vase peut être rapprochée d'une autre représentée sur une mosaïque trouvée dans une maison voisine en Reg. I, ins. 12, 4. Un urceus accompagné d'une inscription y est représenté : "Corinthus fecit »(NSC 1914, 202).

Le parallèle est particulièrement intéressant et il est légitime de penser qu'il s'agit d'un seul et même potier. Nous pouvons donc avancer qu'un

6 Les neufs boutiques mentionnées sont les suivantes : Reg. I, ins. 5,1; Reg. I, ins. 8,10; Reg. III, ins. 1,5 ; Reg. III, ins. 4,1a ; Reg. VII, ins. 4,3 ; Reg. VII, ins. 4,21; Reg. VIII, ins. 4,40-40a; Reg. VIII, ins. 5,8-14 et Reg. IX, ins. 11,4. fabricant d'urceus du nom de Corinthus se trouvait à Pompéi. Eschebach mentionne aussi les deux ateliers susmentionnés (Reg. I, ins. 20, 3 et Reg. II, ins. 3, 7-9) ainsi qu'une officina en Reg. VI, ins. 2,7 dont l'interprétation est incertaine et une autre en Reg. VII, ins. 2,46. Cette dernière, fouillée en 1862, a livré plus de 104 couvercles identiques, mais aucune structure liée à la production (Eschebach 1993, p. $80-81,91-92,157,263)$.

\section{Conclusion}

Nous ne connaissons avec certitude à l'heure actuelle que six structures de productions liées à des fours, dont seulement quatre présentent une documentation suffisante pour permettre un rapprochement entre l'activité du potier et sa production $^{7}$. Dans une fourchette chronologique de quatre siècles, deux fours du $\mathrm{III}^{\mathrm{e}}-\mathrm{II}^{\mathrm{e}}$ siècle avant notre ère, ont produit des amphores et de la céramique à vernis noir, des tuiles et de grands conteneurs, un four du ${ }^{\text {er }}$ siècle de notre ère ayant cuit des pots en céramique commune et un four qui fonctionnait en 79 de notre ère pour la cuisson de lampes à huile et de petits vases.

Ilmanqueunélémentcomplémentaireàlaconnaissance et la compréhension de ces productions que seules les analyses pétrographiques pourraient nous aider à compléter : l'argile. M. Annecchino signale des gisements d'argile dans les niveaux antérieurs à la domus de Salluste (Reg. VI, ins.2,3) ${ }^{8}$ et dans le jardin de la domus du navire Europa (Reg. II, ins.16) ${ }^{9}$. Il semble que l'argile provenant des deux gisements ait la même composition. C'est la preuve que le sous-sol de Pompéi offre des possibilités d'approvisionnement en matière première.
7 Nous ne connaissons pas avec précision quelle est la (ou les) production(s) des fours mis au jour le long de la via dei sepolcri au XIX ${ }^{\mathrm{e}}$ siècle.

8 Annecchino 1977, p. 107. Ce banc d'argile a été mis au jour lors de sondages stratigraphiques réalisés par l'American Academy in Rome en 1971-72.

9 Annecchino 1977, p. 107. Ce gisement aurait identifié par W. Jashemski en 1972. 


\title{
La production de céramique commune à Pompéi Studio petrografico sui reperti ceramici
}

\author{
Celestino Grifa*, Vincenzo Morra**
}

\section{Premessa}

Nell'ambito delle ricerche condotte dal Centre Jean Bérard, undici frammenti di scarti di fornace (da LCP1 a LCP 11) sono stati analizzati al fine di una caratterizzazione mineralogica, petrografica e chimica. In questo contributo sono riportati, in via preliminare, solo i risultati delle osservazioni in microscopia ottica eseguite su sezioni sottili con microscopio ottico polarizzatore (Leitz Laborlux 12 POL ingrandimenti 20x, 40x e 100x).

Le tessiture sono descritte seguendo la terminologia proposta da maggetti (Maggetti 1992) e la stima del grado di addensamento (espresso in $\%$ di superficie) degli inclusi è stata eseguita con l'ausilio delle tabelle di comparazione (Terry, Chilingar 1955).

Le forme analizzate sono quelle descritte nel testo precedente, i precisi riferimenti archeologici sono riportati nella tabella (tab. 1).

\section{Descrizione degli impasti}

\section{LCP1}

Il campione mostra una matrice di colore marrone, otticamente isotropa, con pori e fratture allungati secondo il verso della lavorazione.

L'impasto evidenzia una tessitura seriata con granulometria del degrassante compresa fra 150 e $400 \mu \mathrm{m}$, la percentuale di degrassante è pari

\footnotetext{
* Dipartimento di Studi Geologici ed Ambientali, Università del Sannio, Benevento

** Dipartimento Scienze della terra, Università Federico II, Napoli
}

al 20\%. Si denota l'assenza di inclusi residuali dell'argilla (scheletro), gli inclusi non plastici sono infatti rappresentati solo dal degrassante vulcanico costituito da cliropirosseni, sanidino, biotite e una ulteriore componente vulcanica di scorie e piccoli frammenti di lava.

\section{LCP2}

L'impasto è composto da una matrice marrone scuro con una leggera zonatura cromatica, che individua una porzione centrale dalle tonalità più scure. Ai nicols incrociati la matrice non mostra attività ottica ; la porosità è scarsa.

Gli inclusi non plastici (presenti per il 25\% della superficie totale) si organizzano in una tessitura bimodale con una porzione residuale in cristalli di quarzo e feldspati dalle piccole dimensioni (circa $50 \mu \mathrm{m}$ ) ed una più grossolana porzione di degrassante (150-400 $\mu \mathrm{m})$.

Anche per questo campione il degrassante è rappresentato da materiale vulcanico cristallino (clinopirosseno, sanidino e biotite) e non cristallino (scorie).

\section{LCP3}

Il frammento mostra in sezione sottile una matrice di colore marrone debolmente anisotropa ed una scarsa porosità. La tessitura bimodale evidenzia una frazione di inclusi più fine con cristalli di quarzo e feldspati ed un degrassante subangolare ben classato $(150-400 \mu \mathrm{m})$ composto da clinopirosseni, scorie e pomici. L'addensamento totale degli inclusi è del $25 \%$. 


\begin{tabular}{|l|l|l|l|l|l|}
\hline Campione & US & Classe & Forma & Datazione & Disegno \\
\hline LCP1 & 13.23 & Comune Italica & Di Giovanni 2311a & I a.C-I d.C & 25 \\
\hline LCP2 & 13.24 & Comune Italica & Di Giovanni 2311a & I a.C-I d.C & \\
\hline LCP3 & 13.23 & Comune Italica & Coperchio Y & & 27 \\
\hline LCP4 & 13.06 & Scarto & Distanzatore & & 40 \\
\hline LCP5 & 13.13 & Comune & Gasperetti 1362a & I a.C-I d.C & 18 \\
\hline LCP6 & 13.00 & Scarto & Anello Distanzitore & & 41 \\
\hline LCP7 & 13.00 & Scarto & Anello Distanzitore & & 44 \\
\hline LCP8 & 13.00 & Scarto & Anello Distanzitore & & 45 \\
\hline LCP9 & 13.24 & Scarto & Anello Distanzitore & & 46 \\
\hline LCP10 & 13.23 & Laterizio & & & \\
\hline LCP11 & 13.23 & Laterizio & & & \\
\hline
\end{tabular}

Tab. 1

\section{LCP4}

Questo campione mostra una particolare colorazione della matrice ed organizzazione degli inclusi non plastici a bande. La matrice presenta delle bande più chiare con abbondante presenza di inclusi microcristallini di quarzo, feldspati e muscovite. Si contrappongono a queste delle bande di colore più scuro in cui si concentra il degrassante vulcanico, subangolare e ben calibrato (100-200 $\mu \mathrm{m})$.

Questo particolare tipo di struttura potrebbe rilevare l'utilizzo di due differenti argille miscelate in differenti proporzioni (mixing) per migliorarne le caratteristiche di plasticità e quindi lavorabilità.

\section{LCP5}

La matrice è di colore rosso-arancio leggermente zonata ed isotropa; l'impasto presenta una tessitura bimodale degli inclusi con un addensamento del $20 \%$. I minerali residuali dalle dimensioni di circa $20 \mu \mathrm{m}$ sono costituiti da quarzo e feldspati, il degrassante ancora da clinopirosseno, scorie, sanidino ed in aggiunta piccoli cristalli di granato.

\section{LCP6}

Questo frammento mostra una matrice di colore marrone scuro, inattiva ed una struttura seriata con assenza di inclusi residuali. La porzione non plastica è infatti rappresentata solo dai cristalli dalle dimensioni variabili tra 50 e $200 \mu \mathrm{m}$ di clinopirosseno, sanidino, granato, biotite e scorie.

\section{LCP7}

L'impasto mostra una matrice di colore marrone scuro otticamente isotropa, una tessitura bimodale con addensamento degli inclusi del $20 \%$. Cristalli di quarzo, feldspati e rare lamelle di muscovite sono relegati nella porzione più fine dell'impasto, mentre il degrassante più grossolano, ben classato e subangolare $(50-200 \mu \mathrm{m})$ in cristalli di clinopirosseno e sanidino con piccole scorie.

\section{LCP8}

Questo campione mostra un impasto molto depurato con una matrice di colore marrone chiaro, otticamente inattiva. La tessitura è ancora bimodale ma l'addensamento degli inclusi è inferiore rispetto ai campioni in precedenza descritti (circa il $2 \%$ ).

La scarsa presenza di materiale vulcanico (clinopirosseno, sanidino e scorie, 100-200 $\mu \mathrm{m}$ ) dona all'impasto una tessitura molto fine, sono infatti rilevate maggiori quantità finissimi di inclusi residuali (quarzo, feldspato e muscovite) dalle dimensioni di circa $20 \mu \mathrm{m}$.

\section{LCP9}

Anche in questo campione si evidenzia un impasto molto depurato, sono visibili inclusi microcristallini di quarzo, feldspati e mica bianca accompagnati da una scarsa presenza di degrassante vulcanico, comunque ben classato (dimensioni 100-200 $\mu \mathrm{m}$ ) e subangolare. La matrice di colore marrone chiaro è otticamente isotropa. 


\section{LCP10}

Questo frammento di laterizio presenta la maggior percentuale di inclusi vulcanici (fino al 30\%) e l'assenza di inclusi residuali dell'argilla. Lo smagrante è composto, insieme a fasi precedentemente descritte (clinopirosseno, sanidino, biotite e granato) anche da scorie a composizione modale leucititica e cristalli di olivina.

I grani subangolari possono raggiungere dimensioni di circa $1 \mathrm{~mm}$ con tessitura seriale partendo da dimensioni di $100 \mu \mathrm{m}$, la matrice e marrone scuro, otticamente isotropa.

\section{LCP11}

Campione dalle caratteristiche tessiturali e mineralogiche assimilabili al precedente, entrambi rappresentano frammenti di laterizio.

\section{Discussioni e conclusioni}

I primi dati ricavati dai frammenti di scarti di fornace di epoca romana hanno permesso di puntualizzare le caratteristiche mineralogiche e tessiturali degli impasti.

Per tutti i frammenti è riconoscibile l'utilizzo di un degrassante vulcanico di origine locale come testimoniano la presenza di clinopirosseno calcico nelle due varietà incolore (diopside) e verde scuro (salite), sanidino, biotite, talvolta accompagnati da granato ed olivina nei frammenti di laterizio.

Tutte queste fasi mineralogiche sono riconducibili all'attività del Somma-Vesuvio (es. Joron et al. 1987), insieme alla presenza di scorie a leucite, minerale tipico dell'attività di questo complesso vulcanico non riscontrabile nelle attività degli altri, vicini complessi vulcanici del Golfo di Napoli (Campi Flegrei ed Ischia, es. Melluso et al. 1995 e Vezzoli 1988).

In via preliminare è possibile, altresì ipotizzare l'utilizzo di differenti argille, un'abbondante presenza di inclusi sialici (LCP2, 3, 5, 7, 8 e 9), l'altra contraddistinta dalla totale assenza degli stessi (LCP1, 6, 10 e 11).

Dalle osservazioni in sezione sottile sembra che le due tipologie di argille coesistano nel frammento LCP4 in un processo di mixing. Queste osservazioni dovranno comunque essere confermate da un più approfondito riscontro analitico.

Le analisi petrografiche sugli 11 frammenti analizzati hanno permesso di formare dei gruppi dalle caratteristiche ottiche omogenee.

Un primo gruppo è formato dai campioni LCP1 e LCP6, dal punto di vista formale e funzionale questi rappresentano un'olla ed un distanziatore (vedi testo precedente) realizzati entrambi con argilla senza inclusi sialici con abbondante degrassante vulcanico (tessitura seriata).

I tre frammenti di ceramica comune LCP2 (olla) LCP3 (coperchio) ed LCP5 (brocca), insieme al distanziatore circolare LCP7 vanno ad identificare il gruppo 2, questi frammenti presentano un impasto a tessitura bimodale e abbondante (25\%) degrassante ancora vulcanico (tessitura bimodale).

Anche se tutti i frammenti presentano dal punto di vista tessiturale lo stesso impasto è da notare un colore della matrice variabile all'interno del gruppo, dal rosso-arancio per LCP5 al marrone scuro con zonature scure per LCP2.

I due distanziatori circolari, caratterizzati da un impasto molto depurato con scarsa presenza di inclusi formano il gruppo 3.

L'impasto a tessitura più grossolana, in cui si rileva anche la presenza di grossi cristalli di olivina e scorie con leucite, è specifico per i due frammenti di laterizio (gruppo 4).

Ai 4 gruppi descritti non è possibile associare il frammento LCP4 che sembra evidenziare l'utilizzo di due argille diverse.

Ulteriori analisi di laboratorio permetteranno di definire meglio i gruppi identificati solo in base alle caratteristiche ottiche e inoltre sarà possibile rilevare tutte le altre peculiarità tecnologiche di questi importanti reperti (ad es. temperature di cottura, tipo di argilla utilizzata). 
